\title{
The coexistence of autoimmune rheumatic diseases and thymomas
}

\author{
Ying Jiang ${ }^{A, B, D}$, Xiaodan Dai ${ }^{B}, C$, Liping Duan ${ }^{B, E}$, Yaou Zhou ${ }^{A, F}$ \\ Department of Rheumatology and Clinical Immunology, Xiangya Hospital, Central South University, Changsha, Hunan, China \\ A - research concept and design; B - collection and/or assembly of data; C - data analysis and interpretation; \\ $D$ - writing the article; $E$ - critical revision of the article; $F$ - final approval of article
}

\section{Address for correspondence \\ Yaou Zhou}

E-mail:yaouzhou2015@sina.com

\section{Funding sources}

none declared

Conflict of interest

none declared

Received on September 25, 2015

Revised on 0ctober 26, 2015

Accepted on December 15, 2015

\begin{abstract}
Background. Autoimmune rheumatic diseases (ARDs), involving immune disturbances resulting from auto-inflammatory mechanisms, are a group of diseases characterized by autoimmunity and autoimmunemediated organ damage. Thymoma, whose mechanism is also associated with immune abnormalities, is the most common neoplasm of the anterior mediastinum. But thymoma with ARDs is relatively less frequent. The clinical characteristics of the coexistence of ARDs and thymomas are still not very clear. And the therapeutic strategy for ARDs combined with thymomas varies, with an uncertain outcome.
\end{abstract}

Objectives. The aim of this study was to investigate the clinical characteristics of the coexistence of ARDs and thymomas in order to speculate whether a thymectomy is effective for ARDs combined with thymomas, and to seek the proper therapeutic strategy for treating ARDs combined with thymomas.

Material and methods. We presented 2 cases of the coexistence of ARDs and thymomas. Then, we summarized 20 cases (including our 2 cases) in which the ARD was diagnosed concurrently with, or prior to, the thymoma.

Results. Pure red cell aplastic anemia (PRCA) might be associated with an ARD and a thymoma, and a thymectomy may lead to the appearance, exacerbation, or remission of ARDs.

Conclusions. Searching for a thymoma is necessitated if a patient with ARDs experiences PRCA and the effects of thymectomy in ARDs combined with thymomas may be associated with the onset sequence of ARDs and thymomas.

Key words: autoimmune rheumatic diseases, thymoma, PRCA, thymectomy

DOI

10.17219/acem/61004

\section{Copyright}

Copyright by Author(s)

This is an article distributed under the terms of the

Creative Commons Attribution Non-Commercial License

(http://creativecommons.org/licenses/by-nc-nd/4.0/) 
Autoimmune rheumatic diseases (ARDs) are a group of rare, heterogeneous disorders characterized by autoimmunity and autoimmune-mediated organ damage. They affect an estimated 7.6-9.4\% of the population worldwide, and they include systemic lupus erythematosus (SLE), Sjögren's syndrome (pSS), rheumatoid arthritis (RA), dermatomyositis (DM), and systemic sclerosis (SSc), etc. ${ }^{1}$ Thymoma is the most common tumor in the anterior mediastinum. ${ }^{2}$ However, the combination of an ARD and a thymoma in the same patient is relatively less frequent. Herein, we will present 2 ARD cases that also simultaneously had a thymoma.

\section{Case 1}

A 60-year-old Chinese woman was diagnosed with SLE in 2005 at another hospital because she had polyarthritis, alopecia, neutropenia, and low complete $\mathrm{C} 3 / \mathrm{C} 4$ levels, along with positive antinuclear antibody and anti-dsDNA antibody tests. She was $1^{\text {st }}$ treated with prednisone (50 mg/day), wilfordii (60 mg/day), and methotrexate $(10 \mathrm{mg} /$ week), and her maintenance treatment included prednisone (10 mg/day) for a long time while her disease was stable. She was admitted to our hospital in March 2014 because of dizziness, fatigue, and mild shortness of breath that had persisted for 3 months. A physical examination revealed a pale appearance and mild lower extremity edema. Laboratory findings were as follows: hemoglobin, $30 \mathrm{~g} / \mathrm{L}$; ANA, positive, (with a titer of 1 : 160; granular pattern); anti-dsDNA antibody, negative; anti-histone antibody and antiribosomal protein antibodies, positive; complement C3 and C4, $483.0 \mathrm{mg} / \mathrm{L}$ and $55.3 \mathrm{mg} / \mathrm{L}$, respectively; and Coombs and Ham tests, negative. The albumin level was $30.9 \mathrm{~g} / \mathrm{L}$. A bone marrow cytology test performed in another hospital was as follows: active bone marrow hyperplasia; granulocyte prolifera-

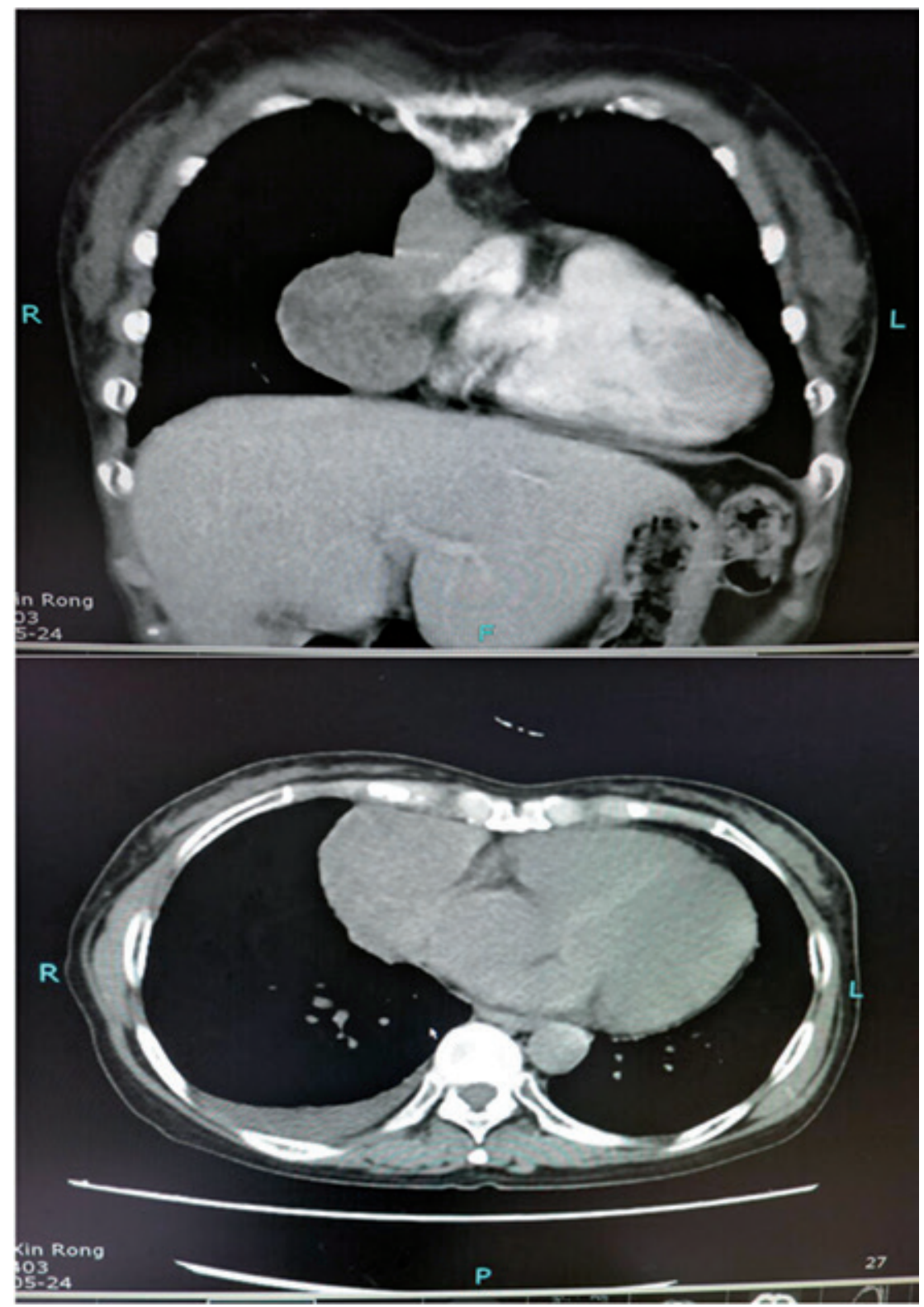

Fig. 1. Computed tomography of the chest showing a mass in the anterior mediastinum (case 1) 

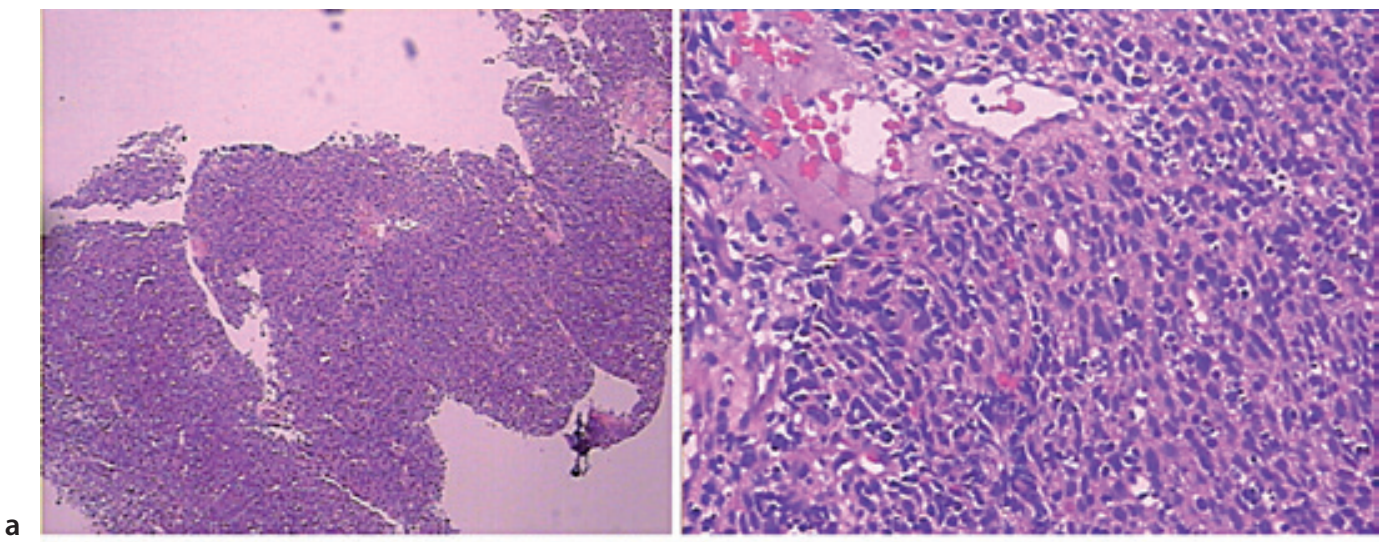

Fig. 2. a) Low-power photomicrograph of the thymic mass (H\&E, $\times 40)$; b) middle-power photomicrograph of the thymic mass ( $H \& E, \times 200)$; c) higherpower photomicrograph of the thymic mass $(H \& E, \times 400)$

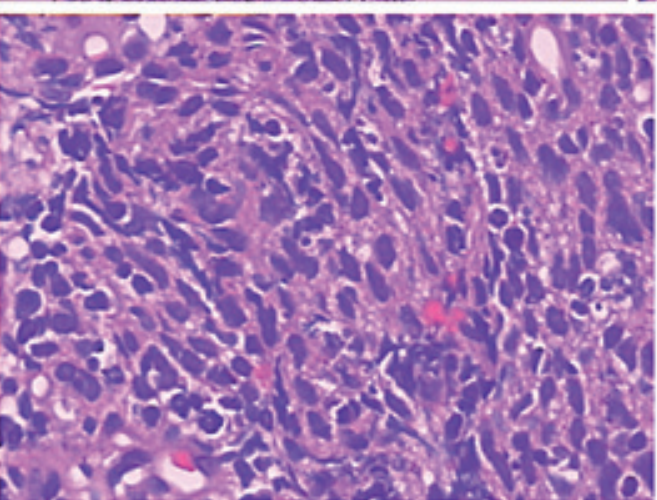

(case 1)

tion; and significantly reduced red blood cell hyperplasia. The megakaryocytes were normal and the platelets were distributed in clusters. The peripheral blood slides were normal. Pure red cell aplastic anemia (PRCA) was diagnosed. A chest computed tomography (CT) scan revealed a mass in the right anterior mediastinum that measured $68 \mathrm{~mm} \times 62 \mathrm{~mm}$ (Fig. 1), with a right pleural effusion. A CT-guided percutaneous needle biopsy of the mass was performed on March 28. The pathological result showed a type B3 thymoma (Fig. 2), according to the World Health Organization (WHO) classification. A thymectomy was not performed because she had severe anemia. She was treated as follows: methylprednisolone (40 mg/day), hydroxychloroquine $(0.2 \mathrm{~g} /$ day $)$, and regular blood transfusions. Hydroxychloroquine (0.2 g/day) and regular blood transfusions were continued, and for daily maintenance, methylprednisolone was gradually reduced to $12 \mathrm{mg}$. Upon her follow-up, her disease was deemed to be stable.

\section{Case 2}

A 63-year-old Chinese woman with a history of hypothyroidism was diagnosed with Sjögren's syndrome in 2007 at our hospital based on dry mouth, dry eyes, positive antinuclear antibody and anti-SSA/SSB tests, and positive corneal fluorescein staining, along with a tear film breakup time of $3 \mathrm{~s}$. Anti-Sm antibody and anti-dsDNA antibody tests were both negative. A urinalysis was negative for proteinuria, and a chest radiograph was normal at that time. She was treated with methylprednisolone $(40 \mathrm{mg} /$ day), wilfordii (60 mg/day), leflunomide (10 mg/day), and levothyroxine $(100 \mu \mathrm{g} /$ day $)$ for her illness. This treatment protocol was continued, and for daily maintenance, methylprednisolone was gradually reduced to $4 \mathrm{mg}$. The disease was stable during follow-up. The patient was admitted to our hospital again because of lower limb edema on August 19, 2014.

Upon admission, a physical examination revealed 4 dental caries with a residual root only and lower extremity edema. The laboratory findings were as follows: blood cell counts, normal; proteinuria, $3+; 24$-h urinary protein, $3.43 \mathrm{~g}$; globulin, $51.60 \mathrm{~g} / \mathrm{L}$; albumin, $27.50 \mathrm{~g} / \mathrm{L}$; triglyceride, $1.58 \mathrm{mg} / \mathrm{L}$; cholesterol, $6.27 \mathrm{mmoL} / \mathrm{L}$; ANA titer, 1 : 320 (homogeneous pattern); anti-SSA and anti-SSB tests, positive; and anti-Sm antibody and anti-dsDNA antibody tests, negative. A chest $\mathrm{CT}$ revealed a large mass measuring $39 \mathrm{~mm} \times 31 \mathrm{~mm}$ in the area of the thymus (Fig. 3).

A thymectomy was performed on August 26, 2014. A type B3 thymoma was considered according to the WHO classification, and the tumor did not involve any other structures (Fig. 4). More than 1 month after the thymectomy, the patient was admitted to our hospital again because of exacerbation of lower extremity edema; the laboratory values were as follows: Blood cell counts, normal; proteinuria, 3+; globulin, $28.60 \mathrm{~g} / \mathrm{L}$; albumin, $24.08 \mathrm{~g} / \mathrm{L}$; triglycerides, $1.58 \mathrm{mg} / \mathrm{L}$; cholesterol, $7.70 \mathrm{mmoL} / \mathrm{L}$; ANA titer, 1 : 320 (homogeneous pattern); 


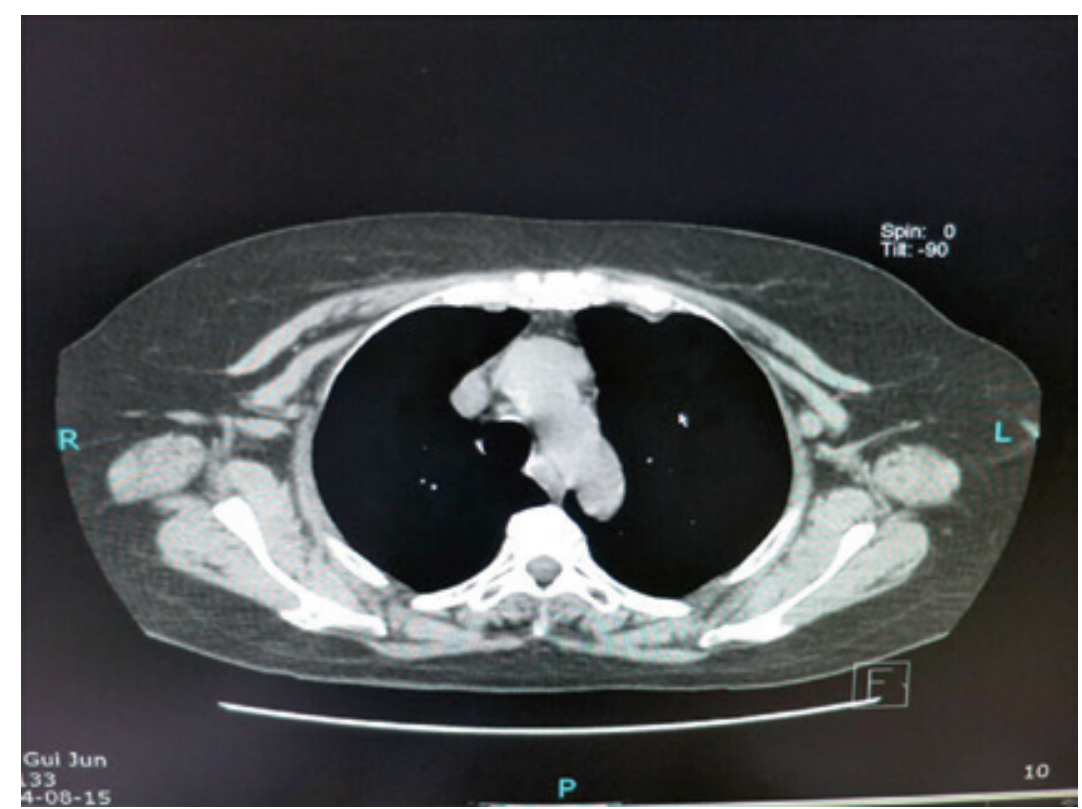

Fig. 3. Computed tomography of the chest showing a mass in the right anterior mediastinum (case 2)
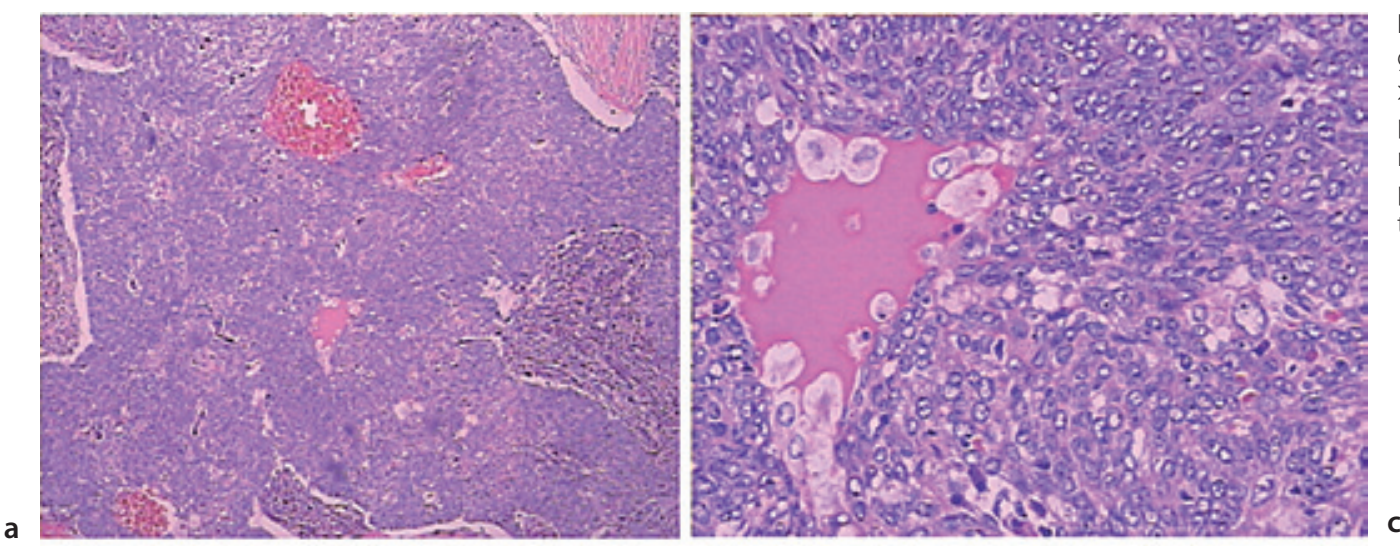

Fig. 4. a) Low-power photomicrograph of the thymic mass ( $H$ \& E, $\times 40)$; b) middle-power photomicrograph of the thymic mass ( $H \& E, \times 200)$; c) higherpower photomicrograph of the thymic mass ( $H \& E, \times 400)$ (case 2)

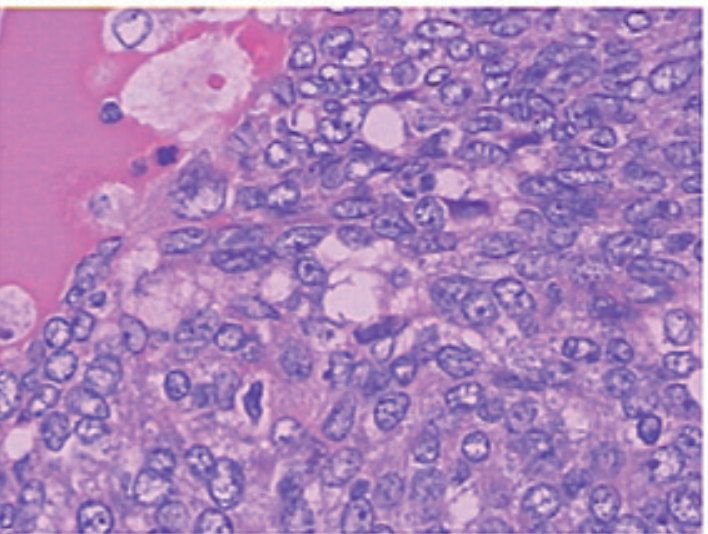

and anti-SSA and anti-SSB tests, still positive. A color Doppler ultrasound revealed thromboses in the external iliac vein, right femoral vein, and popliteal vein. Then, anticoagulant and thrombolytic therapy was administered (low-molecular-weight heparin calcium [4100u BID ip], urokinase [20wu QD IV gtt], and argatroban [20 mg BID IV gtt]). The lower extremity edema gradually subsided. After that, the patient was treated with rivaroxaban (10 mg QD po) as a maintenance anticoagulant treatment.

\section{Discussion}

ARDs are a group of diseases involving immune disturbances resulting from auto-inflammatory mechanisms that are frequently the underlying cause. ${ }^{1}$ A thymoma is the most frequent tumor of the thymus, the mechanism of which is also associated with immune abnormalities. ${ }^{2}$ Structural and functional changes in the thymus may lead to the loss of self-tolerance and may occur with autoim- 
mune diseases. ${ }^{2}$ There are 3 morphological types of thymomas based on genetic alterations according to 2004 WHO classifications: A, B (B1, B2, and B3), and AB. Carcinoma of the thymus is designated as type $C$. Compared with type $\mathrm{A}, \mathrm{B} 1, \mathrm{~B} 2$, and $\mathrm{AB}$, the $\mathrm{B} 3$ subtype has high 15 -year recurrence rates and a poor prognosis. ${ }^{3} \mathrm{~A}$ thymoma is commonly associated with a variety of systemic and autoimmune disorders such as PRCA, hypogammaglobulinemia, pancytopenia, collagen diseases, and most commonly, myasthenia gravis (MG); sometimes 2 or even 3 autoimmune diseases coexist. ${ }^{4}$

In recent years, there have been a few case reports regarding the coexistence of ARDs and thymomas, such as SLE, RA, DM, SSc, and pSS. ${ }^{5-20}$ Several reports documented the appearance of ARD after a thymectomy for a thymoma. Other reports described the appearance of a thymoma years after a patient was diagnosed with an ARD, as in our 2 cases. In some cases, the 2 diagnoses were concurrent. Herein, we have summarized 20 cases (including our 2 cases) and have included relatively complete information. For all 20 cases, the ARD was diagnosed concurrently with or prior to the thymoma (Table 1).

The association of a thymoma with an ARD is not frequent. In clinical studies, the prevalence of SLE in patients with a thymoma varied between 1.5 and $2 \% .{ }^{16}$ PRCA has been associated with a thymoma or ARD. Approximately $2-5 \%$ of patients with a thymoma will have PRCA. ${ }^{21}$ PRCA could also arise as a complication of an ARD such as SLE, but it is very rare. ${ }^{22,23}$ However, the coexistence of all 3 disorders - an ARD, a thymoma, and PRCA - in the same patient is extremely rare. To our knowledge, there have been 6 previous reports of cases with an ARD, a thymoma, and PRCA.12,19,24-27 However, for only 2 of them, an ARD was diagnosed concurrently or prior to a thymoma and PRCA. ${ }^{12,19}$ Herein, we reported on a case (case 1) of a patient with the SLE-thymoma-PRCA triad. The patient developed PRCA and a thymoma 9 years after being diagnosed with SLE. All the patients in the 3 cases were females aged 48, 60, and 63 years, and they had SLE for 3, 9, and 9 years, respectively. Therefore, PRCA might be associated with an ARD and a thymoma, particularly in older patients who have an ARD disease course with a long duration. We speculate that PRCA may be associated with a thymoma, although there is no significant relationship between the occurrence of PRCA and the pathological type of thymoma. Searching for a thymoma is necessitated if a patient with ARD experiences PRCA.

The therapeutic strategy for ARDs combined with thymomas varies, with an uncertain outcome. Underlying conditions involving ARDs and thymomas should be treated. A thymectomy or gamma irradiation of the thymus gland may be performed for a thymoma. Neither chemotherapy nor irradiation is beneficial for the treatment of ARDs. ${ }^{8,28}$ The immunologic effects of a thymec- tomy for an ARD are also not clear. Several reports have documented the appearance, exacerbation, or remission of an ARD after a thymectomy. ${ }^{15}$ Of all the cases reviewed in this report, almost all 9 patients who were diagnosed ARD and a thymoma at the same time achieved remission through either surgery or radiation..$^{8-11,13-16,18}$ In one of these cases, the patient with pSS was deemed to be in remission with surgery alone. ${ }^{18}$ However, for the patients who were diagnosed with an ARD before the thymoma, none achieved remission with surgery alone. Medicines such as corticosteroids, cyclophosphamide, or cyclosporine should also be used to treat ARDs. In spite of this, most patients experience an exacerbation, or their disease becomes refractory to treatment. ${ }^{6,7,11,12}$ As for our $1^{\text {st }}$ case, the patient did not undergo surgery, and her SLE and PRCA were deemed stable. As for our $2^{\text {nd }}$ case, the patient's condition worsened secondary to massive proteinuria and vein thrombosis several months after the thymectomy. It has also been reported that patients with SLE, a thymoma, and PRCA died of a pulmonary embolism after having a thymectomy. ${ }^{27}$ Therefore, a thymectomy or radiation would be effective for helping patients achieve remission with an ARD if the ARD were concurrently diagnosed with a thymoma; however, if the ARD were diagnosed prior to the thymoma, then in addition to treatment for the thymoma, a corticosteroid or immunological inhibitor should be used to help obtain remission for the ARD. Sometimes rituximab, donor lymphocyte infusion (DLI), or hematopoietic stem cell transplantation (HSCT) could also be used to treat the complication, PRCA. ${ }^{19,29}$ We surmise that if a thymoma and an ARD occur simultaneously, the thymoma might exert a role in the development of ARDs, as seen with MG. A thymectomy or radiation might be helpful for achieving remission with an ARD. If the thymoma were to occur several years after the ARD, it would not be clear if the thymoma was the primary abnormality or the immunologic abnormalities seen in patients with an ARD would lead to thymus disorders and tumor development. A thymectomy or radiation is not always associated with remission from an ARD and might sometimes cause an exacerbation. It is necessary to study additional cases to elucidate the effects of thymectomy in ARDs combined with thymomas and to help provide additional guidance for treatment.

In conclusion, we reported 2 cases and compiled a series of cases involving an ARD combined with a thymoma. The data indicated that ARDs can coexist with thymomas and that a thymoma should be included in the differential diagnosis if a patient with an ARD complains of PRCA. The result of a thymectomy for a patient with an ARD can be unpredictable. Indeed, ARDs involve a multisystem disease with heterogeneous and varied manifestations. Therefore, study of additional cases is necessitated for exploring issues related to ARDs and thymomas. 


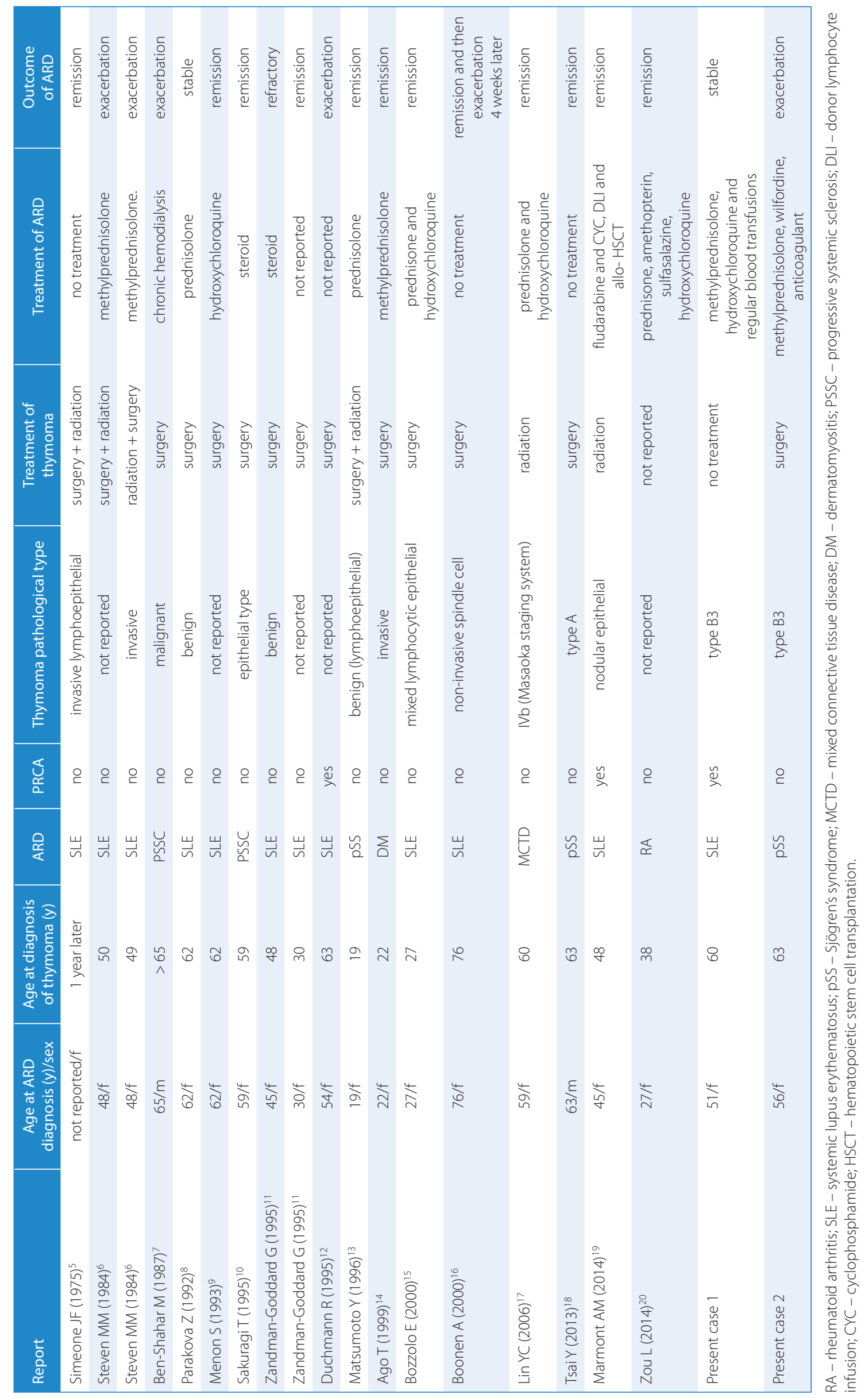




\section{References}

1. Goldblatt F, O'Neill SG. Clinical aspects of autoimmune rheumatic diseases. Lancet. 2013;382(9894):797-808.

2. Shelly S, Agmon-Levin N, Altman A, Shoenfeld Y. Thymoma and autoimmunity. Cell Mol Immunol. 2011;8(3):199-202.

3. Strobel $P$, et al. Thymoma and thymic carcinoma: An update of the WHO Classification 2004. Surg Today. 2005;35(10):805-811.

4. Mejri N, Chabchoub I, Gargouri I. Effect of chemotherapy on autoimmune hepatitis in thymoma: A case report and literature review. Cancer Biol Med. 2013;10(3):169-173.

5. Simeone JF, McCloud T, Putman CE, Marsh J. Thymoma and systemic lupus erythematosus. Thorax. 1975;30(6):697-700.

6. Steven MM, Westedt ML, Eulderink F, Hazevoet HM, Dijkman JH, Cats A. Systemic lupus erythematosus and invasive thymoma: Report of two cases. Ann Rheum Dis. 1984;43(6):825-828.

7. Ben-Shahar M, Rosenblatt E, Green J, Cohen I. Malignant thymoma associated with progressive systemic sclerosis. Am J Med Sci. 1987, 294(4), p. 262267.

8. Parakova Z, Hrncir Z, Nozicka Z. Thymoma in the anterior mediastinum and systemic lupus erythematosus. Vnitr Lek. 1992;38(2):177-183.

9. Menon S, Snaith ML, Isenberg DA. The association of malignancy with SLE: An analysis of 150 patients under long-term review. Lupus. 1993;2(3):177-181.

10. Sakuragi T, Furukawa T, Yonemura T, Yoshida T, Irie K. Thymoma associated with progressive systemic sclerosis: A case report. Nihon Kyobu Geka Gakkai Zasshi. 1995;43(1):135-138.

11. Zandman-Goddard G, Lorber M, Shoenfeld Y. Systemic lupus erythematosus and thymoma: A double-edged sword. Int Arch Allergy Immunol. 1995;108(1):99-102.

12. Duchmann R, Schwarting A, Poralla T, Meyer zum Büschenfelde KH Hermann E. Thymoma and pure red cell aplasia in a patient with systemic lupus erythematosus. Scand J Rheumatol. 1995;24(4):251-254.

13. Matsumoto $Y$, Hirai S, Ohashi M, Imaizumi M. Sjogren's syndrome associated with thymoma. J Am Acad Dermatol. 1996;35(4):639-640.

14. Ago T, Nakamura M, Iwata I, et al. Dermatomyositis associated with invasive thymoma. Intern Med. 1999;38(2):155-159.

15. Bozzolo E, Bellone M, Quaroni N, Voci C, Sabbadini MG. Thymoma associated with systemic lupus erythematosus and immunologic abnormalities. Lupus. 2000;9(2):151-154.

16. Boonen A, Rennenberg R, van der Linden S. Thymoma-associated systemic lupus erythematosus, exacerbating after thymectomy. A case report and review of the literature. Rheumatology (Oxford). 2000;39(9):1044-1046.

17. Lin YC, Wu CC, Ou TT, Yen JH, Liu HW, Tsai WC. Malignant thymoma associated with mixed connective tissue disease: A case report. Clin Rheumatol. 2006;25(2):262-264.
18. Tsai Y, Lin Y, Chen C, Tzao C. Thymoma associated with myasthenia gravis and Sjogren syndrome. West Indian Med J. 2013;62(3):264-265.

19. Marmont AM, Bacigalupo A, Gualandi F, Bregante S, van Lint MT, Geroldi S. Systemic lupus erythematosus complicated with thymoma and pure red cell aplasia (PCRA): CR of both complications following thymectomy and allogeneic haematopoietic SCT (HSCT), but persistence of antinuclear antibodies (ANA). Bone Marrow Transplant. 2014;49(7):982-983.

20. Zou L, Xiong Z, Dun Y, Xiong Y. A case of rheumatoid arthritis associated with Hashimoto's thyroiditis and thymoma. Int J Rheum Dis. 2014. doi: 10.1111/1756-185X.12453.

21. Chintakuntlawar AV, Rizvi SA, Cassivi SD, Pardanani A. Thymomaassociated pancytopenia: Immunosuppressive therapy is the cornerstone for durable hematological remission. Ann Hematol. 2015; 94(3):453-458.

22. Habib GS, Saliba WR, Froom P. Pure red cell aplasia and lupus. Semin Arthritis Rheum. 2002;31(4):279-283.

23. Koyama, RV, Silva LF, Henriques VB, Tran C, Yoshikawa GT. Pure red cell aplasia associated with systemic lupus erythematosus. Acta Reumatol Port. 2014;39(3):265-268.

24. Fallahi S, Akbarian M, Dabiri S. Pure red cell aplasia as a presenting feature in systemic lupus erythematosus and association with thymoma, hypothyroidism and hypoparathyroidism: A case report and literature review. Iran J Allergy Asthma Immunol. 2014;13(2): 138-143.

25. Mankia S, Giles IP. Pure red cell aplasia in a patient with lupus-like disease and thymoma. Grand Rounds. 2011;11(1):37-40.

26. MacKechnie HL, Squires AH, Platts M, Pruzanski W. Thymoma, myasthenia gravis, erythroblastopenic anemia and systemic lupus erythematosus in one patient. Can Med Assoc J. 1973;109(8):733-738.

27. Iwadate $H$, Kobayashi $H$, Shio $K$, et al. A case of systemic lupus erythematosus complicated by pure red cell aplasia and idiopathic portal hypertension after thymectomy. Mod Rheumatol. 2006;16(2): 109-112.

28. Rosman A, Atsumi T, Khamashta MA, Ames PR, Hughes GR. Development of systemic lupus erythematosus after chemotherapy and radiotherapy for malignant thymoma. Br J Rheumatol. 1995;34(12): 1175-1176.

29. Gupta RK, Ezeonyeji AN, Thomas AS, Scully MA, Ehrenstein MR, Isenberg DA. A case of pure red cell aplasia and immune thrombocytopenia complicating systemic lupus erythematosus: Response to rituximab and cyclophosphamide. Lupus. 2011;20(14):1547-1550. 